\title{
Usefulness of 1-year of thyroid stimulating hormone suppression on additional levothyroxine in patients who underwent hemithyroidectomy with papillary thyroid microcarcinoma
}

\author{
Jin Gu Kang, Young Ah Kim, Jung Eun Choi, Soo Jung Lee, Su Hwan Kang \\ Department of Surgery, Yeungnam University College of Medicine, Daegu, Korea \\ Contributions: (I) Conception and design: JG Kang, YA Kim, JE Choi, SJ Lee, SH Kang; (II) Administrative support: None; (III) Provision of study \\ materials and patients: SH Kang, SJ Lee; (IV) Collection and assembly of data: JG Kang; (V) Data analysis and interpretation: JG Kang; (VI) \\ Manuscript writing: All authors; (VII) Final approval of manuscript: All authors. \\ Correspondence to: Su Hwan Kang. Department of Surgery, Yeungnam University College of Medicine, 170 Hyunchoong-ro, Namgu, Daegu, Korea. \\ Email: kangsuhwan@yu.ac.kr.
}

\begin{abstract}
Background: The purpose of this study was to identify usefulness of 1-year of thyroid stimulating hormone (TSH) suppression, on additional levothyroxine in patients who underwent hemithyroidectomy with papillary thyroid microcarcinoma (PTMC).

Methods: Two-hundred consecutive patients who had received hemithyroidectomy February 2011 to March 2013, were enrolled, retrospectively. Group 1, only, was taking levothyroxine for a year, postoperatively. We evaluated postoperative hypothyroidism through serum TSH level, measured periodically.

Results: Postoperative TSH $>10$ was significantly different, at $13 \%$ and $25 \%$, between two groups $(\mathrm{P}=0.036)$. Twenty patients in group 1, and 32 patients in group 2, received additional levothyroxine. Multivariate analysis showed that 1-year suppression, clinical thyroiditis, and preoperative TSH $>2$, were significantly associated with additional levothyroxine (OR 2.17, $\mathrm{P}=0.025$ and $\mathrm{OR} 2.00, \mathrm{P}=0.046$ and $\mathrm{OR}$ 2.64, $\mathrm{P}=0.006$ ). Too, 1-year TSH suppression, preoperative $\mathrm{TSH}>2$, were also significantly associated with postoperative $\mathrm{TSH}>10(\mathrm{OR} 2.55, \mathrm{P}=0.022$ and $\mathrm{OR} 2.22, \mathrm{P}=0.048)$.

Conclusions: We suggest 1-year TSH suppression after hemithyroidectomy, for PTMC in patients with preoperative $\mathrm{TSH}>2 \mathrm{mU} / \mathrm{L}$ and clinical thyroiditis, to reduce additional levothyroxine.
\end{abstract}

Keywords: Hemithyroidectomy; hypothyroidism; levothyroxine; papillary thyroid microcarcinoma (PTMC); thyroid stimulating hormone (TSH)

Submitted Jun 12, 2019. Accepted for publication Sep 27, 2019.

doi: 10.21037 /gs.2019.10.12

View this article at: http://dx.doi.org/10.21037/gs.2019.10.12

\section{Introduction}

Papillary thyroid microcarcinoma (PTMC), is defined as a PTC tumor less than $1 \mathrm{~cm}$. We supposed that the proportion of PTMC has been increasing gradually, because of a complex reason such as ultrasonography development, and easy accessibility to examination. Fortunately, PTMC was almost regarded as low risk of recurrence (2.4\%), and less than $1 \%$ risk of distant metastasis. According to the $8^{\text {th }}$ edition of American Joint Committee on Cancer (AJCC), and 2015 American Thyroid Association (ATA) guidelines, minor extrathyroidal extension (ETE) was categorized to T1 stage, and they recommend hemithyroidectomy for a tumor less than $4 \mathrm{~cm}$. Although central lymph node metastasis was incidentally identified by prophylactic central neck dissection (pCND), they generally recommend hemithyroidectomy, followed by mild TSH suppression therapy (TSH target $0.1-0.5 \mathrm{mU} / \mathrm{L}$ ), then complete thyroidectomy $(1,2)$. On this trend, hemithyroidectomy as standard treatment for PTMC, will be in a strong position, regardless of central lymph node metastasis. 
Prognosis is favorable in hemithyroidectomy patients with low risk PTMC, and TSH suppression is not usually recommended in low risk patients. But we could consider using additional levothyroxine after hemithyroidectomy in selected patients, because current guidelines recommend TSH $0.5-2 \mathrm{mU} / \mathrm{L}$ range for low risk PTMC. On different perspectives, it was easy to maintain thyroid function after hemithyroidectomy because of the remnant lobe. However, unlike the theory, we have observed that incidence of subclinical hypothyroidism after hemithyroidectomy was surprisingly high, and even permanent thyroid hormone replacement, was required in some cases. Although hemithyroidectomy, postoperative hypothyroidism and symptoms could occur, and sometimes require life-long thyroid hormone replacement. Many previous studies have had an interest in risk factors for hypothyroidism, but such factors remain unknown. Additionally, these studies reported that postoperative hypothyroidism is mostly subclinical, and that patients recover spontaneously, in many cases. Relatively well-known risk factors for postoperative hypothyroidism have been mentioned, such as preoperative TSH level, thyroiditis, and anti-thyroid peroxidase (TPO) antibody (3-7).

However, we focused that usefulness of 1-year TSH suppression on additional levothyroxine after hemithyroidectomy, through incidence of meaningful hypothyroidism $(\mathrm{TSH}>10)$, and symptoms that required permanent thyroid hormone replacement. To our knowledge, there has been no study, on TSH suppression's direct effect on incidence of hypothyroidism. The purpose of this study is to identify the usefulness of one year of TSH suppression on hypothyroidism, and recurrence which required additional levothyroxine, in patients who underwent hemithyroidectomy with PTMC.

\section{Methods}

\section{Patient}

Two-hundred consecutive patients who received hemithyroidectomy for PTMC, by two endocrine surgeons at Yeungnam University Medical Center February 2011 to March 2013, were enrolled retrospectively. This study was approved by the Institutional Ethics Review Board (201905-021). All patients were followed at least for 5 years and examined via laboratory test yearly. Inclusion criteria were as follow: (I) PTC with tumor less than $1 \mathrm{~cm}$, proven by histology, (II) not pre-existing hypothyroidism, (III) laboratory test annually for at least 5 years, (IV) follow-up more than 5 years. Two-hundred patients were divided into two groups, according to 1-year TSH suppression. Group 1 equally took levothyroxine (Synthyroxine ${ }^{\circledR} 100 \mathrm{mcg}$, levothyroxine sodium, Northwind Pharmaceuticals, LLC) during 1 year postoperatively, to suppress TSH level within 0.5-2.0 mU/L (suppression group). Group 2 did not take levothyroxine during follow-up (no suppression Group). Serum TSH level was measured periodically in order with preoperative, postoperatively 6 months, and then annually up to 5 years. Laboratory tests such as T3, FT4, thyroglobulin ( $\mathrm{Tg}$ ), and $\mathrm{Tg}$ antibody, were examined as the same method. Anti TPO antibody, was checked preoperatively once. Chest $\mathrm{X}$-ray and ultrasonography were examined once a year. Also, we evaluated other clinicohistological factors for developing hypothyroidism (TSH >10) as well as symptoms and identified correlation of additional levothyroxine.

\section{Hypothyroidism}

Postoperative hypothyroidism was defined as serum TSH level $>3.8 \mathrm{mU} / \mathrm{L}$, and subclinical hypothyroidism was defined as high TSH level, without symptoms. First, we checked incidence, of subclinical hypothyroidism and recurrence. Second, meaningful hypothyroidism $(\mathrm{TSH}>10)$ as well as symptoms, were identified in each group. Third, we also wanted to identify predictive factors to additional levothyroxine, through incidence of hypothyroidism (TSH $>10$ ) as well as symptoms. The receiver operating characteristics curve (ROC) was used, to identify cut off value of preoperative TSH level, as predictive modality.

\section{Statistical analysis}

Continuous variables were expressed, as mean \pm standard deviation (SD) by Student's $t$-test. Chi-square test and Fisher's exact test were used, to compare categorical variables, and then expressed by number and percentage. Multivariate analyses were performed, by logistic regression analysis. All statistical analyses were performed, using SPSS version 21.0 (SPSS, Inc., Chicago, IL, USA). And P values $<0.05$ were statistically significant.

\section{Results}

Two-hundred patients consisted of 173 females and 27 males. Mean age was $42.79 \pm 9.60$ and $45.43 \pm 8.90(\mathrm{P}=0.045)$, follow-up was $83.75 \pm 15.29$, and $83.22 \pm 15.54$ in each group. Preoperative subclinical hypothyroidism was $3 \%$ and $7 \%$ 
Table 1 Patient characteristics between two groups

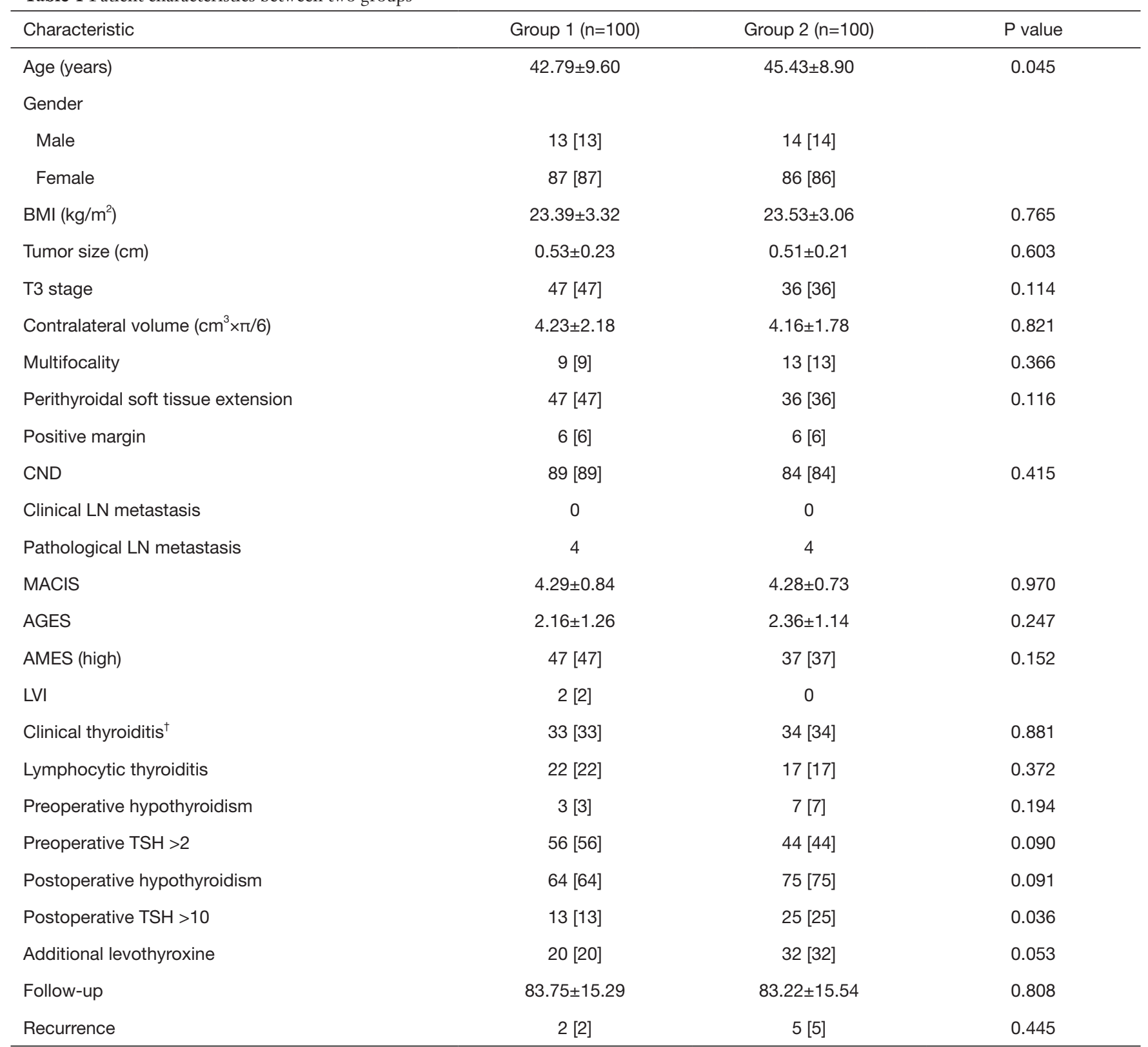

Values are presented as mean \pm standard deviation or number [\%]. ${ }^{\dagger}$, diffuse thyroiditis or anti-TPO antibody positivity or Tg antibody positivity. BMI, body mass index; CND, central neck dissection; LN, lymph node; MACIS, metastasis, age, completeness of resection, invasion and size; AGES, age, tumor grade, extent and size; AMES, age, metastasis, extent and size; LVI, lymphovascular invasion; TSH, thyroid stimulating hormone; TPO, thyroperoxidase; Tg, thyroglobulin.

$(\mathrm{P}=0.194)$, respectively. By contrast, postoperative subclinical hypothyroidism was quite high (64\% vs. $75 \%, \mathrm{P}=0.091)$, and recurrence was $2 \%$ and $5 \%(\mathrm{P}=0.445)$, respectively. Clinical thyroiditis (anti-TPO antibody, Tg antibody and diffuse thyroiditis) was $33 \%$ and $34 \%(\mathrm{P}=0.881)$, additional levothyroxine was $20 \%$ and $32 \%(\mathrm{P}=0.053)$ and preoperative
TSH $>2$ was $56 \%$ and $44 \%(\mathrm{P}=0.090)$, respectively. There was no significant difference, in most of values between the two groups. However, postoperative TSH $>10$ was significantly different as $13 \%$ and $25 \%$ between two groups $(\mathrm{P}=0.036)$, respectively. Not only that, but other clinicohistological factors are summarized in Table 1. 
Table 2 Additional levothyroxine cases in each group

\begin{tabular}{lcc}
\hline Cause & $\begin{array}{c}\text { Group 1 } \\
(20 / 24)\end{array}$ & $\begin{array}{c}\text { Group 2 } \\
(32 / 35)\end{array}$ \\
\hline Exclusion & 1 & \\
Fatigue (normal TSH) & 1 & 3 \\
Recurrence (normal TSH) & 2 & \\
Pregnancy & 2 & 2 \\
TSH 5-10 (U/mL) & 2 \\
General weakness & 1 & 1 \\
Fatigue & & 1 \\
Indeterminate LN (positive margin) & & \\
Recurrence & 4 & 1 \\
Stage III (N1a) with headache & 13 & 25 \\
Stage III (T3 or N1a) & & \\
TSH >10 (U/mL) & & \\
Recurrence & & \\
\hline
\end{tabular}

TSH, thyroid stimulating hormone; LN, lymph node. T3, minimal extrathyroidal extension by AJCC 7th; $\mathrm{N} 1 \mathrm{a}$, metastasis level $\mathrm{VI}$ or VII.

Twenty-four patients in group 1 received additional levothyroxine, but four patients (one fatigue alone with normal TSH level, one recurrence with normal TSH level, two pregnancies), were excluded among of them. Finally, 20 patients, were composed of $13 \mathrm{TSH}>10$ and 7 TSH 5-10, respectively. And seven patients with TSH 5-10 consisted of two fatigue, one recurrence and four stage III (T3 or N1a). In group 2, 35 patients received additional levothyroxine, but three patients (three recurrence with normal TSH level) were excluded. Thirty-two patients consisted of $25 \mathrm{TSH}$ $>10$ (one recurrence) and seven TSH 5-10, respectively. Each of the seven patients with TSH 5-10, expressed two general weakness, one stage III (N1a) with headache, two fatigue, one indeterminate LN (positive margin), and one recurrence (Table 2).

Figure 1 shows mean TSH levels in each group during 5 years. Preoperative TSH was of similar value, between the two groups. However, postoperative 6 months and 1-year TSH levels were inevitably different, because of taking a dose of levothyroxine for 1 year. And then, TSH value tended to be similar, between each group. Finally, 5-year mean TSH level was 3.31 and $3.44 \mathrm{mU} / \mathrm{L}$, respectively. There was no difference between the two groups, except for the 1-year TSH suppression period.

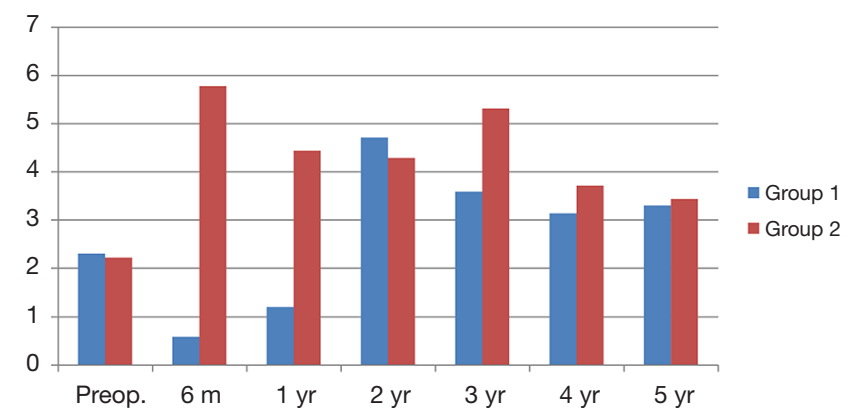

Figure 1 Mean TSH levels in each group during follow-up. TSH, thyroid stimulating hormone.

Using the ROC curve, cut off value of TSH was $2.0 \mathrm{mU} / \mathrm{L}$, as measured by AUC (69\% sensitivity, 56\% specificity, respectively). Univariate analysis shows preoperative TSH $>2$ positivity and clinical thyroiditis, are associated with additional levothyroxine $(\mathrm{OR}=2.63, \mathrm{P}=0.004$ and $\mathrm{OR}=2.34$, $\mathrm{P}=0.010$, respectively). Multivariate analysis shows that 1 year suppression, clinical thyroiditis, and preoperative TSH $>2$, are significantly associated with additional levothyroxine $(\mathrm{OR}=2.17, \mathrm{P}=0.025$ and $\mathrm{OR}=2.00, \mathrm{P}=0.046$ and $\mathrm{OR}=2.64, \mathrm{P}=0.006$ ) (Table 3).

Table 4 shows univariate and multivariate analysis, for postoperative TSH $>10$. In 38 patients who developed postoperative TSH $>10,1$ year TSH suppression and clinical thyroiditis were related with postoperative TSH $>10(\mathrm{OR}=2.28 ; 95 \% \mathrm{CI}, 1.04-5.00, \mathrm{P}=0.036$ and $\mathrm{OR}=$ 2.14; 95\% CI, 1.00-4.57, $\mathrm{P}=0.046$ ), by univariate analysis. Multivariate analysis showed 1-year TSH suppression, preoperative $\mathrm{TSH}>2$, were significantly associated with postoperative $\mathrm{TSH}>10(\mathrm{OR}=2.55, \mathrm{P}=0.022$ and $\mathrm{OR}=2.22$, $\mathrm{P}=0.048)$, respectively.

Figure 2 compares frequency of newly developed postoperative TSH $>10$, during postoperative five years between the two groups. Postoperative TSH $>10$ was found in 13 and 25 patients ( $13 \%$ vs. $25 \%, \mathrm{P}=0.036)$ respectively, and peak incidence of postoperative hypothyroidism was 13 at 6 months in group 1 , and 9 at 2 years in group 2. The shape of two graphs was similar, but incidence and peak time were significantly different, as previously mentioned. In conclusion, this graph was so characteristic, of 1-year TSH suppression effect on postoperative TSH $>10$.

Recurrence were found as $2 \%$ and $5 \%$, respectively, in each group $(\mathrm{P}=0.445)$. Margin positivity $(\mathrm{OR}=5.40 ; 95 \%$ CI, 0.93-1.07, $\mathrm{P}<0.001)$, lymphocytic thyroiditis $(\mathrm{OR}=3.31$; 95\% CI, 0.99-1.01, $\mathrm{P}=0.001)$, and multifocality $(\mathrm{OR}=2.82$; 
Table 3 Univariate and multivariate analysis for additional levothyroxine

\begin{tabular}{|c|c|c|c|c|c|c|}
\hline Variable & \multicolumn{3}{|c|}{ Univariate } & \multicolumn{3}{|c|}{ Multivariate } \\
\hline 1-year TSH suppression & 1.88 & $0.99-3.59$ & 0.053 & 2.17 & $1.10-4.28$ & 0.025 \\
\hline Age ( $>45$ years) & 1.23 & $0.65-2.32$ & 0.527 & & & NA \\
\hline Gender (female) & 0.61 & $0.22-1.70$ & 0.341 & & & NA \\
\hline Contralateral volume $\left(\mathrm{cm}^{3} \times \pi / 6\right)$ & & & 0.993 & & & NA \\
\hline Clinical thyroiditis $^{\dagger}$ & 2.34 & $1.22-4.48$ & 0.010 & 2.00 & $1.01-3.94$ & 0.046 \\
\hline Lymphocytic thyroiditis & 1.15 & $0.53-2.51$ & 0.726 & & & NA \\
\hline Preoperative TSH >2.0 (U/mL) & 2.63 & $1.35-5.11$ & 0.004 & 2.64 & $1.31-5.29$ & 0.006 \\
\hline
\end{tabular}

${ }^{\dagger}$, anti-TPO antibody positivity, Tg antibody positivity, diffuse thyroiditis. OR, odds ratio; $\mathrm{Cl}$, confidence interval; TSH, thyroid stimulating hormone; NA, not applicable; BMI, body mass index; TPO, thyroperoxidase; Tg, thyroglobulin.

Table 4 Univariate and multivariate analysis for postoperative TSH $>10$

\begin{tabular}{|c|c|c|c|c|c|c|}
\hline Variable & \multicolumn{3}{|c|}{ Univariate } & \multicolumn{3}{|c|}{ Multivariate } \\
\hline 1 year TSH suppression & 2.28 & $1.04-5.00$ & 0.036 & 2.55 & $1.14-5.69$ & 0.022 \\
\hline Age ( $>45$ years) & 1.52 & $0.71-3.26$ & 0.279 & & & NA \\
\hline Gender (female) & 0.37 & $0.082-1.63$ & 0.171 & & & NA \\
\hline Contralateral volume $\left(\mathrm{cm}^{3} \times \pi / 6\right)$ & & & 0.850 & & & NA \\
\hline Clinical thyroiditis $^{\dagger}$ & 2.14 & $1.00-4.57$ & 0.046 & & & NA \\
\hline Lymphocytic thyroiditis & 1.14 & $0.45-2.85$ & 0.786 & & & NA \\
\hline Preoperative $\mathrm{TSH}>2.0(\mathrm{U} / \mathrm{mL})$ & 1.95 & $0.90-4.22$ & 0.086 & 2.22 & $1.01-4.90$ & 0.048 \\
\hline
\end{tabular}

${ }^{\dagger}$, clinical thyroiditis, anti-TPO antibody positivity, Tg antibody positivity, diffuse thyroiditis. TSH, thyroid stimulating hormone; OR, odds ratio; $\mathrm{Cl}$, confidence interval; NA, not applicable; BMI, body mass index; TPO, thyroperoxidase; Tg, thyroglobulin.

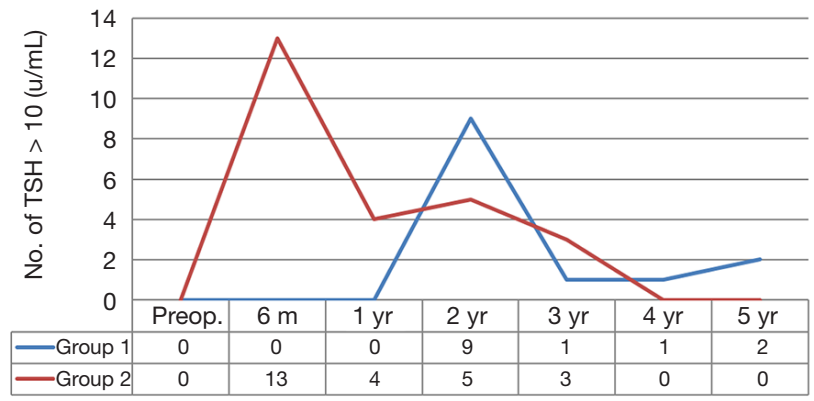

Figure 2 Frequency of newly developed postoperative TSH $>10$ annually in each group. TSH, thyroid stimulating hormone.
95\% CI, 0.936-1.068, $\mathrm{P}=0.005$ ) were statistically significant for recurrence (Table 5).

\section{Discussion}

In this study, 200 patients were enrolled retrospectively, February 2011 to March 2013. Minimal extrathyroidal extension (ETE) was categorized to T3 according to AJCC $7^{\text {th }}$ and our institution uses $0.23-3.8 \mathrm{mU} / \mathrm{L}$, as normal TSH range. The major exclusion of this study was pre-existing hypothyroidism, because several previous studies commonly 
Table 5 Multivariate analysis for recurrence

\begin{tabular}{lccc}
\hline Recurrence & OR & $95 \% \mathrm{Cl}$ & P value \\
\hline Margin positivity & 5.40 & $0.93-1.07$ & $<0.001$ \\
Lymphocytic thyroiditis & 3.31 & $0.99-1.01$ & 0.001 \\
Multifocality & 2.82 & $0.94-1.07$ & 0.005 \\
\hline
\end{tabular}

OR, odds ratio; $\mathrm{Cl}$, confidence interval.

reported preoperative hypothyroidism, as predictive factors for postoperative hypothyroidism. In this study, postoperative hypothyroidism means TSH $>3.8 \mathrm{mU} / \mathrm{L}$, without symptoms. According to the 2015 ATA guidelines, they recommend no TSH suppression (TSH target $0.5-2 \mathrm{mU} / \mathrm{L}$ ), with low risk PTC. However, in cases of no TSH suppression, post hemithyroidectomy was more frequent, above the $0.5-2.0 \mathrm{mU} / \mathrm{L}$ range. In our study, T3 (minimal extrathyroidal extension) was 47 and 36, six margin positivity and four central lymph node metastases in each group. In group 2, maintaining target TSH $0.5-2 \mathrm{mU} / \mathrm{L}$ was observed as $6 \%$ at postoperative 6 months, $10 \%$ at 1 year, $13 \%$ at 2 years. We used levothyroxine (Synthyroxine $100 \mathrm{mcg}$ ) to reduce risk of recurrence and maintain adequate TSH level.

Hemithyroidectomy has been standard treatment, for low risk PTMC. According to AJCC 8th and 2015 ATA guidelines, minimal ETE was newly revised, to T1 and low risk was defined as follow: (I) absence of local or distant metastasis, (II) macroscopic tumor resection completely, (III) no tumor invasion of loco-regional tissues or structures, (IV) No aggressive histology, (V) no vascular invasion, (VI) clinical N0 or $\leq 5$ pathologic N1 micrometastases $(<0.2 \mathrm{~cm}$ in largest dimension) (1). Recently, clinicians prefer follow up with or without mild TSH suppression, than completion of thyroidectomy in case of a small volume of central lymph node metastasis. As mentioned above, for various reasons, standard treatment for PTMC regardless of N1a, weights on hemithyroidectomy.

It is generally thought that PTMC leads to excellent prognosis, without TSH suppression. Some studies have reported risk of locoregional recurrence is $2.4 \%$, although frequently, lymph node metastasis with PTMC and risk of disease-specific mortality and distant metastasis, were $0 \%$ and less than $1 \%(8)$. Other studies have reported mortality rate of low risk PTC was $1 \%$, but recurrence rate was relatively high at $8 \%(9)$. Too, recurrence rate in the remnant thyroid gland was $2.6 \%$ at 10 years, and regional lymph node recurrence was $5.4 \%$ at 10 years (10). As mentioned above, most studies considered risk factors such as ETE, lymphovascular invasion, multifocality, and margin positivity. Hemithyroidectomy with PTMC, mild TSH suppression, can be inevitable in existence of risk factors for recurrence. Our study also shows two and five recurrence in each group, and margin positivity, lymphocytic thyroiditis, and multifocality were significant for recurrence.

Suppression therapy is basic on theory, that thyroid cancer cells typically express thyroid stimulating hormone receptor and binding TSH receptor, with levothyroxine lead to inhibitory effect on cancer growth. Some systemic reviews and meta-analyses show that higher TSH level, is associated with higher OR of thyroid cancer (11). Patients who underwent lobectomy for PTMC, and are maintaining target TSH level (0.5-2 mU/L), may not need levothyroxine (12). There is scant evidence to guide TSH target or use of thyroid hormone, in ATA low risk patients who have undergone lobectomy. By contrast, there is lack of evidence, about TSH suppression effect, on normal thyroid glands. Our study shows lower incidence and delayed peak time, of postoperative TSH $>10$ in the 1 -year TSH suppression group. This implies that TSH suppression, has specific influence on normal thyroid tissue. Theoretically, remnant lobe has adequate thyrocytes, to maintain normal thyroid function after lobectomy $(13,14)$. However, our study shows loss of supplementation cause to elevated TSH for 1 year, and then TSH level gradually recovered. It was probably caused by remnant thyroid lobe recovery, as well as adaptation to poor circumferences. Suppressed thyroid lobe has minimalized function during 1 year. It is like cryopreservation of thyrocytes. That leads to immediate evasion of surgical stress during the postoperative period, and restarts the function to maintain normal thyroid, after full recovery the body. We can explain the effect of 1-year TSH suppression, from this evasion phenomenon. Of course, although suppressed thyroid began to work, the function could be somewhat short of the remnant lobe. If this hypothesis is correct, preoperative thyroid function is a very important factor in the occurrence of postoperative hypothyroidism, and it can also be a useful predictive factor.

There is a considerable number of postoperative hypothyroidism after hemithyroidectomy, including subclinical hypothyroidism. In our study, subclinical hypothyroidism was found at $64 \%$ and $75 \%$, respectively in each group, although many spontaneously recover. Too, postoperative $\mathrm{TSH}>10$ which required levothyroxine replacement, was found at $13 \%$ and $25 \%$, respectively. In group 2, incidence was relatively high, in comparison 
to previous studies. One study reported prevalence of hypothyroidism reached $64 \%$ in patients who underwent hemithyroidectomy, and $68 \%$ patients among of them spontaneously recovery. Also, higher serum TSH level $(>1.7 \mathrm{mU} / \mathrm{L})$ was an independent risk factor, for hypothyroidism (15). Several studies also show that approximately one third of euthyroid patients who underwent hemithyroidectomy developed hypothyroidism, and the significant factor, was commonly preoperative TSH. Postoperative hypothyroidism was known, as most occurred in less than 1 year (16-19). One study shows that more than $90 \%$ of cases developed hypothyroidism within the first 6 months, and $0.8 \%$ of hypothyroidism developed after more than 1 year (20). Paradoxically, to evade TSH stimulation during 1 year postoperatively, may be critical to low incidence of hypothyroidism.

Limitations of our study were retrospective analysis with a small cohort, and short follow-up. First, selection bias could have existed, because we excluded preexisting hypothyroidism, and same number of patients in each group. Second, although we set a target TSH $0.5-2 \mathrm{mU} / \mathrm{L}$, taking a synthyroxine 100 mcg equally in group 1, led to lower TSH than target range in some patients. Third, duration and timing of TSH suppression lacked evidence, and minimal ETE was categorized to $\mathrm{T} 3$ according to AJCC 7th unlike now.

Additionally, it is quite difficult to predict cases of additional levothyroxine, because of several reasons. Fortunately, postoperative hypothyroidism, accounts for a large portion. Despite the above limitations, our study has a special meaning in terms of 1-year TSH suppression's direct effect, on postoperative hypothyroidism.

\section{Conclusions}

Unlike pre-existing studies, our study shows high incidence of postoperative subclinical hypothyroidism (64\% vs. $75 \%$ ), and postoperative TSH >10 (13\% vs. $25 \%$, $\mathrm{P}=0.036)$. Univariate and multivariate analysis show that 1-year suppression, preoperative $\mathrm{TSH}>2$ and clinical thyroiditis, were significantly associated with additional levothyroxine $(\mathrm{OR}=2.17, \mathrm{P}=0.025$, $\mathrm{OR}=2.64, \mathrm{P}=0.006$; $\mathrm{OR}=2.00, \mathrm{P}=0.046)$. Also, 1 -year TSH suppression and preoperative $\mathrm{TSH}>2$ were significantly associated with postoperative $\mathrm{TSH}>10(\mathrm{OR}=2.55, \mathrm{P}=0.022$ and $\mathrm{OR}=2.22$, $\mathrm{P}=0.048$ ). Recurrence was associated with margin positivity, lymphocytic thyroiditis, and multifocality. But not 1-year TSH suppression alone $(\mathrm{OR}=5.40 ; 95 \%$ CI, 0.93-1.07,
$\mathrm{P}<0.001$ and $\mathrm{OR}=3.31 ; 95 \% \mathrm{CI}, 0.99-1.01, \mathrm{P}=0.001$ and $\mathrm{OR}=2.82 ; 95 \% \mathrm{CI}, 0.94-1.07, \mathrm{P}=0.005)$. In conclusion, we suggest 1-year TSH suppression after hemithyroidectomy for PTMC in patients, with preoperative TSH $>2 \mathrm{mU} / \mathrm{L}$ and clinical thyroiditis, to reduce additional levothyroxine.

\section{Acknowledgments}

None.

\section{Footnote}

Conflicts of Interest: The authors have no conflicts of interest to declare.

Ethical Statement: The authors are accountable for all aspects of the work in ensuring that questions related to the accuracy or integrity of any part of the work are investigated and resolved. This study was approved by the Institutional Ethics Review Board of Yeungnam University College of Medicine (No. 2019-05-021).

\section{References}

1. Ullmann TM, Gray KD, Stefanova D, et al. The 2015 American Thyroid Association guidelines are associated with an increasing rate of hemithyroidectomy for thyroid cancer. Surgery 2019;166:349-55.

2. Walczyk A, Kopczynski J, Gasior-Perczak D, et al. Poorly differentiated thyroid cancer in the context of the revised 2015 American Thyroid Association Guidelines and the Updated American Joint Committee on Cancer/TumorNode-Metastasis Staging System (eighth edition). Clin Endocrinol (Oxf) 2019;91:331-9.

3. Johner A, Griffith OL, Walker B, et al. Detection and management of hypothyroidism following thyroid lobectomy: evaluation of a clinical algorithm. Ann Surg Oncol 2011;18:2548-54.

4. Koh YW, Lee SW, Choi EC, et al. Prediction of hypothyroidism after hemithyroidectomy: a biochemical and pathological analysis. Eur Arch Otorhinolaryngol 2008;265:453-7.

5. Ng P, Ho C, Tan WB, et al. Predictors of thyroxine replacement following hemithyroidectomy in a south east Asian cohort. Head Neck 2019;41:1463-7.

6. Stoll SJ, Pitt SC, Liu J, et al. Thyroid hormone replacement after thyroid lobectomy. Surgery 2009;146:554-8; discussion 8-60. 
7. Verloop H, Louwerens M, Schoones JW, et al. Risk of hypothyroidism following hemithyroidectomy: systematic review and meta-analysis of prognostic studies. J Clin Endocrinol Metab 2012;97:2243-55.

8. Yoo JY, Stang MT. Current Guidelines for Postoperative Treatment and Follow-Up of Well-Differentiated Thyroid Cancer. Surg Oncol Clin N Am 2016;25:41-59.

9. Ebina A, Sugitani I, Fujimoto Y, et al. Risk-adapted management of papillary thyroid carcinoma according to our own risk group classification system: is thyroid lobectomy the treatment of choice for low-risk patients? Surgery 2014;156:1579-88; discussion 88-9.

10. Matsuzu K, Sugino K, Masudo K, et al. Thyroid lobectomy for papillary thyroid cancer: long-term followup study of 1,088 cases. World J Surg 2014;38:68-79.

11. McLeod DS, Watters KF, Carpenter AD, et al. Thyrotropin and thyroid cancer diagnosis: a systematic review and dose-response meta-analysis. J Clin Endocrinol Metab 2012;97:2682-92.

12. McLeod DS, Sawka AM, Cooper DS. Controversies in primary treatment of low-risk papillary thyroid cancer. Lancet 2013;381:1046-57.

13. Ito M, Miyauchi A, Kang S, et al. Effect of the presence of remnant thyroid tissue on the serum thyroid hormone balance in thyroidectomized patients. Eur J Endocrinol

Cite this article as: Kang JG, Kim YA, Choi JE, Lee SJ, Kang SH. Usefulness of 1-year of thyroid stimulating hormone suppression on additional levothyroxine in patients who underwent hemithyroidectomy with papillary thyroid microcarcinoma. Gland Surg 2019;8(6):636-643. doi: 10.21037/ gs.2019.10.12
2015;173:333-40.

14. Lang BH, Wong CKH, Wong KP, et al. Effect of Thyroid Remnant Volume on the Risk of Hypothyroidism After Hemithyroidectomy: A Prospective Study. Ann Surg Oncol 2017;24:1525-32.

15. Park S, Jeon MJ, Song E, et al. Clinical Features of Early and Late Postoperative Hypothyroidism After Lobectomy. J Clin Endocrinol Metab 2017;102:1317-24.

16. Chotigavanich C, Sureepong P, Ongard S, et al. Hypothyroidism after Hemithyroidectomy: The Incidence and Risk Factors. J Med Assoc Thai 2016;99:77-83.

17. Lee DY, Seok J, Jeong WJ, et al. Prediction of thyroid hormone supplementation after thyroid lobectomy. J Surg Res 2015;193:273-8.

18. Said M, Chiu V, Haigh PI. Hypothyroidism after hemithyroidectomy. World J Surg 2013;37:2839-44.

19. Ha TK, Kim DW, Park HK, et al. The Effect of Levothyroxine Discontinuation Timing on Postoperative Hypothyroidism after Hemithyroidectomy for Papillary Thyroid Microcarcinoma. Int J Endocrinol 2016;2016:3240727.

20. Tomoda C, Ito Y, Kobayashi K, et al. Subclinical hypothyroidism following hemithyroidectomy: a simple risk-scoring system using age and preoperative thyrotropin level. ORL J Otorhinolaryngol Relat Spec 2011;73:68-71. 